\title{
SÍNDROME DE WEST
}

\section{EVOLUÇÃO CLÍNICA E ELETRENCEFALOGRÁFICA DE 70 PACIENTES E RESPOSTA AO TRATAMENTO COM HORMÔNIO ADRENOCORTICOTRÓFICO, PREDNISONA, VIGABATRINA, NITRAZEPAM E ÁCIDO VALPRÓICO}

\author{
SÉRGIO A. ANTONIUK*, ISAC BRUCK*, ADRIANE SPESSATTO**, SILVIA M. HALICK**, \\ LAIS R. DE BRUYN**, EDUARDO MEISTER***, DUILTON DE PAOLA****
}

\begin{abstract}
RESUMO - Em estudo retrospectivo avaliamos a evolução clínica e eletrencefalográfica das formas criptogênica e sintomática da síndrome de West e analisamos a eficácia do hormônio adrenocorticotrófico, vigabatrina, prednisona, ácido valpróico e nitrazepam no controle dos espasmos. Participaram do estudo 70 pacientes, acompanhados por período maior que 2 anos. Doze (17\%) eram criptogênicos e 58 (83\%) sintomáticos. O grupo criptogênico apresentou percentagem significativamente maior de pacientes que frequentavam escola regular e desenvolvimento motor normal, melhor controle das crises epilépticas, tendência menor a evoluir para síndrome de Lennox Gastaut e $83,3 \%$ tiveram controle completo dos espasmos (72,4\% dos pacientes do grupo sintomático obtiveram controle completo dos espasmos). O hormônio adrenocorticotrófico e a vigabatrina foram as drogas mais eficazes, controlando $68,75 \%$ e $60 \%$ dos espasmos, respectivamente, quando utilizados como droga de primeira escolha e $75 \%$ e $50 \%$, respectivamente, como drogas de segunda escolha.
\end{abstract}

PALAVRAS-CHAVE: síndrome de West, espasmos infantis, epilepsia, tratamento, hormônio adrenocorticotrófico, prednisona, vigabatrina, ácido valpróico e nitrazepam.

West syndrome: clinical and eletroencephalographic follow up of 70 patients and response to the adrenocorticotropic hormone, prednisone, vigabatrin, nitrazepam and valproate

\begin{abstract}
In a retrospective study we assessed the outcome of the criptogenic and symptomatic forms of West syndrome and evaluated the efficacy of adrenocorticotropic hormone, vigabatrin, prednisone, valproate and nitrazepam in the spasms control. Seventy patients were follwed up by 2 years. Twelve (17\%) were criptogenics and $58(83 \%)$ symptomatics. In criptogenic group significantly more patients were in regular school classes and with normal motor development, better control of seizure, less tendency to evoluate to Lennox Gastaut syndrome and $83.3 \%$ had control of spasms ( $72.4 \%$ of patients from symptomatic group had control of spasms). Adrenocorticotropic hormone and vigabatrin were the most efective drugs, with $68.75 \%$ and $60 \%$ of spasms control, respectivelly, when used as first line of therapy and $75 \%$ and 50\%, respectivelly, as second line of therapy.
\end{abstract}

KEY WORDS: West syndrome, infantile spasms, epilepsy, treatment, adrenocorticotropic hormone, prednisone, vigabatrin, valproate, nitrazepam.

Os espasmos infantis (EI) foram descritos pela primeira vez em 1841 pelo Dr James West em uma carta à revista Lancet, na qual ele relatou o caso de seu próprio filho, referindo que este possuía "uma forma peculiar de convulsões do lactente"1. Em 1952, Gibbs e Gibbs² descreveram o padrão eletrencefalográfico denominado "hipsarritmia". Um grande marco na história da síndrome de West

*Professores assistentes do Centro de Neurologia Pediátrica (CENEP), Departamento de Pediatria, Hospital de Clínicas da Universidade Federal do Paraná (UFPR): **Médico Residente em Neuropediatria do Hospital de Clínicas UFPR; ***Médico Neuropediatra do Hospital de Clínicas da UFPR; ****Professor Assistente do Serviço de Neurofisiologia do Hospital de Clínicas da UFPR. Aceite: 10-abril-2000.

Dr. Sérgio A. Antoniuk - CENEP - Rua Floriano Essenfelder 81 - 80060-270 Curitiba PR - Brasil. Fax $412649101 / 3629385$. 
(SW) ocorreu em 1958, quando Sorel e Dusaucy-Bauloye sugeriram o uso do hormônio adrenocorticotrófico (ACTH) para o tratamento desta desordem ${ }^{3}$. Desde então, os avanços têm sido cada vez maiores, sendo os mais recentes, do campo da neuroimagem.

Os EI, além de serem um tipo de crise, correspondem a uma síndrome de múltiplas causas. De acordo com a classificação das epilepsias e das síndromes epilépticas da International League Against Epilepsy (ILAE) ${ }^{4}$ a síndrome clássica, também conhecida como síndrome de West é caracterizada por espasmos, atraso ou regressão no desenvolvimento psicomotor e eletrencefalograma (EEG) com hipsarritmia.

O ACTH tem sido o tratamento de escolha desta patologia, contudo, efeitos colaterais frequentes, e algumas vezes graves, têm ocorrido. Outros medicamentos, tais como o ácido valpróico, os benzodiazepínicos, os corticosteróides e a piridoxina têm sido utilizados mas, na maioria dos estudos, a resposta não tem sido comparável à do ACTH. A vigabatrina (VGB) é droga que inibe a GABA transaminase, aumentando os níveis de GABA cerebrais; publicações recentes têm relatado a eficácia desta droga no tratamento da $\mathrm{SW}^{5-8}$, principalmente quando secundária a esclerose tuberosa.

Os objetivos deste estudo foram: analisar a evolução clínica e eletrencefalográfica de 70 pacientes com SW, comparando a forma criptogênica com a sintomática e avaliar a eficácia do ACTH, da VGB, da prednisona (PRE), do ácido valpróico (VPA) e do nitrazepam (NTZ) no controle dos espasmos.

\section{MÉTODO}

Em um estudo retrospectivo foram analisados os dados de prontuários de 70 pacientes com SW acompanhados no Centro de Neuropediatria do Hospital de Clínicas da UFPR no período de junho de 1979 a setembro de 1996.

O desempenho cognitivo foi avaliado nas crianças com idade igual ou superior a 5 anos, por informações obtidas dos pais destes pacientes e através do tipo de escola que frequentavam (escola regular, classe e escola especiais). Para a avaliação do desenvolvimento motor, utilizamos o exame neurológico tradicional de Lefévre ${ }^{9}$ e, através dele, os pacientes foram classificados da seguinte forma: 1) exame neurológico normal; 2) exame neurológico com anormalidades leves (quando foram encontradas alterações discretas do tônus muscular, do equilíbrio estático e dinâmico e da coordenação motora fina); e 3) paralisia cerebral (hemiplégica, tetraplégica, diplégica, distônica e mista).

Foram incluídos os pacientes que preencheram os seguintes critérios de inclusão:

1. Espasmos infantis e atraso ou regressão do desenvolvimento psicomotor;

2. Exame de neuroimagem - tomografia computadorizada (TC) e/ou ressonância magnética (RM) de crânio;

3. EEG compatível com hipsarritmia, de acordo com os critérios de Lombroso ${ }^{10}$ ou hipsarritmia modificada, de acordo com os critérios de Hrachovy et al. ${ }^{11}$;

4. Período mínimo de 2 anos de acompanhamento.

A SW foi classificada segundo a $\mathrm{ILAE}^{4}$ em criptogênica ou sintomática. Os casos que possuem etiologia conhecida, ou sinais de lesão cerebral precedendo o início dos espasmos, são classificados como sintomáticos; aqueles que não possuem etiologia conhecida, ou sinais de lesão cerebral precedendo os espasmos, são classificados como criptogênicos.

Entre os pacientes sintomáticos, as causas são frequentemente agrupadas de acordo com o momento da ocorrência do insulto (pré-natal, perinatal ou pós-natal).

Os pacientes com SW criptogênica foram comparados com os sintomáticos quanto à evolução do desenvolvimento psicomotor, da epilepsia e das alterações eletrencefalográficas.

Também foi avaliada a eficácia do tratamento com ACTH, VGB, PRE, VPA e NTZ como tratamento de primeira escolha no controle dos espasmos. O ACTH foi administrado em uma dose que variou de 20-40 UI e a duração do tratamento de 1-12 meses. A VGB foi administrada na dose de $35-75 \mathrm{mg} / \mathrm{kg} / \mathrm{dia}$, o VPA de $15-60 \mathrm{mg} / \mathrm{kg} /$ dia, o NTZ de 0,5-1 mg/kg/dia e a PRE de 2-5 mg/kg/dia. Se o controle dos espasmos não fosse obtido dentro de período máximo de 4 semanas, a droga era substituída ou outra medicação era adicionada ao esquema terapêutico.

A eficácia dos tratamentos com ACTH, PRE, VPA, VGB e NTZ foi considerada da seguinte forma: 1 excelente (controle completo dos espasmos); 2 - boa (controle $\geq 50 \%$ dos espasmos); e 3 - insatisfatória (controle $<50 \%$ dos espasmos). 
Tabela 1. Etiologia associada à síndrome de West sintomática $(n=58)$.

a) Causas pré-natais:

$15(25,9 \%)$

Esclerose tuberosa

4

Malformações cerebrais

6

Infecções intrauterinas crônicas

3

Fenilcetonúria

1

Síndrome alcoólico fetal

b) Causas perinatais:

$16(27,6 \%)$

Síndrome hipóxico-isquêmica

12

Hipoglicemia

4

c) Causas pós-natais:

$$
\begin{aligned}
& \text { Infecções do SNC } \\
& \text { Pós-imunização } \\
& \text { Distúrbio metabólico }
\end{aligned}
$$

6

2

d) Etiologia desconhecida:

A análise estatística dos resultados foi realizada mediante a utilização do programa Statista, versão 5.0, tendo sido utilizado o teste do qui-quadrado de Pearson (se necessário com a correção de Yates). Considerou-se como nível de significância estatística valor de $\mathrm{p}<0,05$.

\section{RESULTADOS}

O período médio de seguimento dos pacientes foi 6,8 anos, variando de 2 a 19 anos.

Trinta e dois $(45,7 \%)$ pacientes eram do gênero feminino e 38 (54,3\%) do masculino.

A média de idade do início dos espasmos foi 5,3 meses, variando de 2 dias de vida a 22 meses (Fig 1).

Dos 70 pacientes, $12(17 \%)$ foram classificados como criptogênicos e $58(83 \%)$ sintomáticos. Entre os sintomáticos $15(25,9 \%)$ eram secundários a causas pré-natais, $16(27,6 \%)$ perinatais, 9 $(15,5 \%)$ pós-natais e 18 (31\%) de etiologia desconhecida (Tabela 1). Os achados da TC de crânio dos pacientes com SW Sintomática estão resumidos na Tabela 2 e o perfil dos pacientes classificados como portadores de SW sintomática e que apresentaram TC de crânio normal está exposto na Tabela 3.

Na avaliação do desempenho cognitivo de acordo com a escolaridade, observamos que $3(30 \%)$ dos pacientes com SW criptogênica e $2(3,7 \%)$ dos sintomáticos frequentavam escola regular (p < 0,05) (Fig 2).

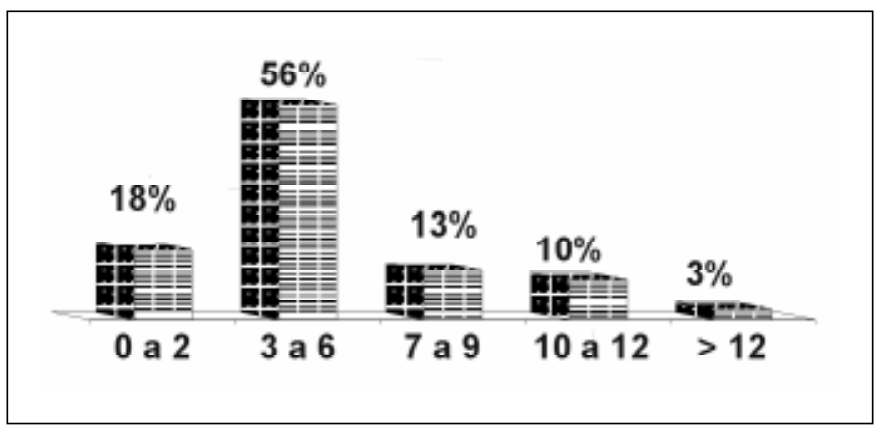

Fig 1. Idade de início dos espasmos (em meses). 
Tabela 2. Achados encontrados nas tomografias de crânio dos pacientes com síndrome de West sintomática $(n=58)$.

Atrofia cerebral

Normal

Compatível com isquemia cerebral

Malformação cerebral

Calcificações subependimárias

Leucomalácia periventricular

Calcificações e atrofia

Imagens hipodensas nos cornos frontais

Calcificações

Malformação cerebral e calcificações múltiplas
$19(32,8 \%)$

$14(24,1 \%)$

$9(15,5 \%)$

$6(10,3 \%)$

$4(7 \%)$

$2(3,5 \%)$

$1(1,7 \%)$

$1(1,7 \%)$

$1(1,7 \%)$

$1(1,7 \%)$

Em relação ao desenvolvimento motor, $60 \%$ dos pacientes com SW sintomática e $25 \%$ dos com SW criptogênica apresentavam paralisia cerebral (p <0,05) (Fig 3).

Quanto à evolução da epilepsia, embora não tenha existido significância estatística, no grupo criptogênico houve tendência maior para o controle completo das crises epilépticas e menor tendência a evoluir para síndrome de Lennox Gastaut. (Fig 4).

Com referência à evolução eletrencefalográfica, 11 (15,7\%) dos 70 pacientes evoluíram com EEG normal. Embora não tenha existido diferença estatística, no grupo criptogênico houve tendência maior para a normalização do EEG (Tabela 4).

Dezesseis pacientes receberam ACTH, 14 PRE, 17 VPA, 5 VGB e 15 NTZ como droga de primeira escolha no tratamento dos espasmos (Tabela 5). Três pacientes utilizaram outras drogas e foram excluídas do estudo. Onze $(68,75 \%)$ dos 16 pacientes tratados com ACTH e $3(60 \%)$ dos tratados com VGB apresentaram controle completo dos espasmos, sem recidiva. Com exceção da VGB, o ACTH foi significativamente mais eficaz que as demais drogas (NTZ, VPA e PRE) no controle dos espasmos. No entanto, quando foi comparada a eficácia da VGB com a das demais drogas no controle dos espasmos, esta foi significativamente mais eficaz apenas em relação à PRE.

Do total, $22(32,8 \%)$ dos 67 pacientes apresentaram controle completo dos espasmos, sem recidiva (6 pacientes com SW criptogênica e 16 com SW sintomática) com o tratamento de primeira

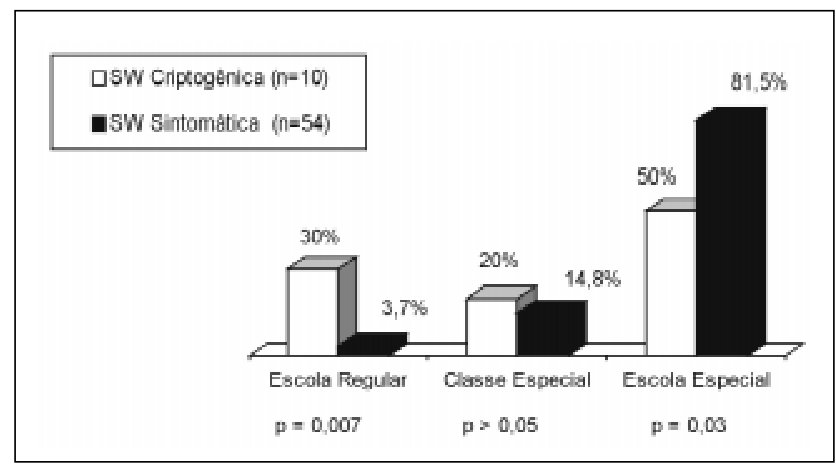

Fig 2. Avaliação cognitiva dos pacientes com idade maior ou igual a 5 anos, de acordo com a escolaridade. 
Tabela 3. Etiologia dos pacientes com síndrome de West sintomática e TC de crânio normal.

\begin{tabular}{cccc}
\hline Etiologia & $\begin{array}{c}\text { DPM* anterior ao } \\
\text { início dos espasmos }\end{array}$ & Exame Neurológico & $\begin{array}{c}\text { Número de } \\
\text { pacientes }\end{array}$ \\
\hline SHI & atrasado & PC tetraespástica & 1 \\
Desconhecida & atrasado & Normal & 6 \\
Desconhecida & atrasado & Normal & 2 \\
Desconhecida & atrasado & PC tetraespástica & 1 \\
Meningite bacteriana & normal & PC tetraespástica & 2 \\
Toxoplasmose congênita & atrasado & PC hemiplégica & 1 \\
Após vacina tríplice & normal & PC hemiplégica & 1 \\
\hline
\end{tabular}

DPM, Desenvolvimento Psicomotor; SHI, Síndrome hipóxico-isquêmica; PC, Paralisia cerebral.

Tabela 4. Evolução eletrencefalográfica da síndrome de West.

\begin{tabular}{ccc}
\hline & $\begin{array}{c}\text { Criptogênica } \\
(\mathrm{n}=12)\end{array}$ & $\begin{array}{c}\text { Sintomática } \\
(\mathrm{n}=58)\end{array}$ \\
\hline Normal* $^{*}$ & $4(33,3 \%)$ & $7(12 \%)$ \\
Difusamente lento, sem \\
anormalidades específicas & $2(16,7 \%)$ & $7(12 \%)$ \\
Hipsarritmia* & 0 & $4(6,9 \%)$ \\
Hipsarritmia modificada* & 0 & $7(12 \%)$ \\
Ponta-onda lenta* & 0 & $6(10,4 \%)$ \\
Atividade irritativa & $2(16,7 \%)$ & $9(15,5 \%)$ \\
Focal* & $2(16,7 \%)$ & $6(10,4 \%)$ \\
Multifocal* & $1(8,3 \%)$ & $6(10,4 \%)$ \\
Generalizada* & $1(8,3 \%)$ & $6(10,4 \%)$ \\
\hline Generalizada e Multifocal* & & \\
\hline
\end{tabular}

$* p>0,05$

Tabela 5. Eficácia do ACTH, vigabatrina (VGB), prednisona (PRE), ácido valpróico (VPA) e nitrazepam (NTZ) como tratamento de primeira escolha no tratamento dos espasmos.

\begin{tabular}{ccccc}
\hline Droga (n) & \multicolumn{3}{c}{ Eficácia } \\
\cline { 2 - 5 } & $\begin{array}{c}\text { Excelente } \\
\text { sem recidiva }\end{array}$ & $\begin{array}{c}\text { Excelente } \\
\text { com recidiva }\end{array}$ & Boa & Insatisfatória \\
\hline ACTH (16) & $11(68,75 \%)$ & $2(12,5 \%)$ & $1(6,25 \%)$ & $2(12,5 \%)$ \\
VGB (5) & $3(60 \%)$ & 0 & 0 & $2(40 \%)$ \\
NTZ (15) & $3(20 \%)$ & $2(13,3 \%)$ & $2(13,3 \%)$ & $8(53,4 \%)$ \\
VPA (17) & $3(17,6 \%)$ & $2(11,8 \%)$ & $1(5,9 \%)$ & $11(64,7 \%)$ \\
PRE (14) & $2(14,3 \%)$ & $1(7,1 \%)$ & $2(14,3 \%)$ & $9(64,3 \%)$ \\
\hline
\end{tabular}




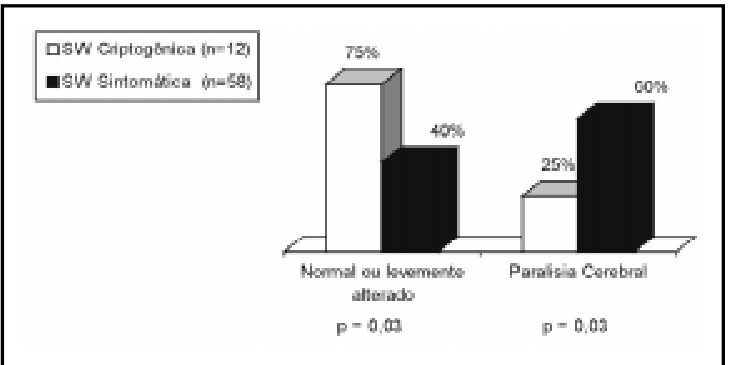

Fig 3. Evolução do desenvolvimento motor.

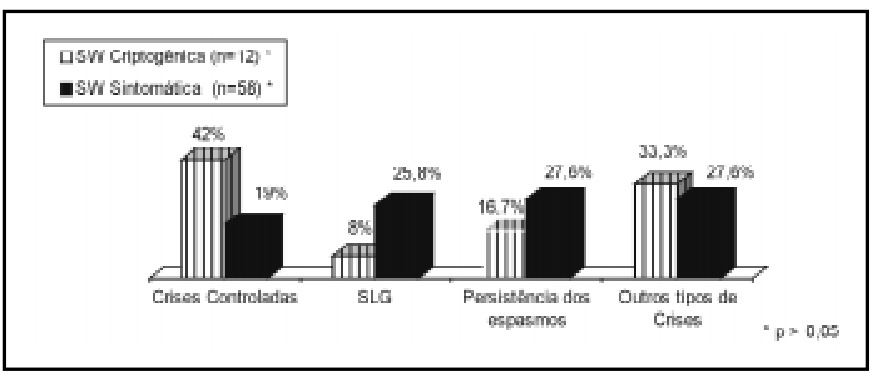

Fig 4. Evolução da epilepsia.

Tabela 6. Eficácia do ACTH, vigabatrina (VGB), prednisona (PRE), ácido valpróico (VPA) e nitrazepam (NTZ) como drogas de seguda escolha no tratamento dos espasmos.

\begin{tabular}{ccccc}
\hline Droga (n) & \multicolumn{3}{c}{ Eficácia } \\
\cline { 2 - 5 } & $\begin{array}{c}\text { Excelente } \\
\text { sem recidiva }\end{array}$ & $\begin{array}{c}\text { Excelente } \\
\text { com recidiva }\end{array}$ & Boa & Insatisfatória \\
\hline ACTH (4) & $3(75 \%)$ & 0 & 0 & $1(25 \%)$ \\
VGB (2) & $1(50 \%)$ & 0 & 0 & $1(50 \%)$ \\
PRE (8) & $1(12,5 \%)$ & $2(25 \%)$ & $3(37,5 \%)$ & $2(25 \%)$ \\
VPA (13) & $1(7,7 \%)$ & $1(7,7 \%)$ & $1(7,7 \%)$ & $10(76,9 \%)$ \\
NTZ (12) & $1(8,3 \%)$ & $1(8,3 \%)$ & $4(33,4 \%)$ & $6(50 \%)$ \\
\hline
\end{tabular}

escolha. Dos 6 pacientes com SW criptogênica que apresentaram controle completo dos espasmos, sem recidiva, 4 responderam ao ACTH, 1 à VGB e 1 ao VPA. Quanto aos 16 pacientes com SW sintomática, 7 responderam ao ACTH, 2 à VGB, 2 à PRE, 3 ao NTZ e 2 ao VPA.

Dos 45 pacientes que apresentaram recidiva dos espasmos ou respostas boa e insatisfatória, 13 receberam VPA, 12 NTZ, 8 PRE, 4 ACTH E 2 VGB como drogas de segunda escolha. Seis pacientes receberam outras drogas e, portanto, foram excluídos da segunda fase da avaliação da eficácia das drogas ( 1 destes pacientes recebeu lamotrigina e teve controle completo dos espasmos, sem recidiva). Como drogas de segunda escolha, embora não tenhamos feito nenhum teste estatístico devido ao pequeno tamanho das amostras, o ACTH e a VGB novamente mostraram tendência a serem mais eficazes que a PRE, o VPA e o NTZ no tratamento dos espasmos (Tabela 6).

Após a utilização de uma segunda droga, apenas 7 (18\%) dos 39 pacientes apresentaram controle completo dos espasmos, sem recidiva. Vinte e dois pacientes foram submetidos a uma terceira droga, 
sendo que nenhum apresentou controle completo dos espasmos sem recidiva e 7 tiveram seus espasmos temporariamente controlados (2 com PRE, 2 com VPA, 2 com NTZ e 1 com ACTH).

Uma quarta droga ainda foi tentada em 7 pacientes (2 receberam ACTH, 2 PRE 2 VPA e 1 VGB), sendo que apenas o que recebeu VGB apresentou $100 \%$ de controle dos espasmos, sem recidiva, evoluindo para a síndrome de Lennox-Gastaut.

\section{DISCUSSÃO}

No presente estudo houve leve predomínio do gênero masculino sobre o feminino $(54,3 \%$ e $45,7 \%$ respectivamente). Este resultado também foi encontrado por Cowan e Hudson ${ }^{12}$.

Em nosso estudo, 12 (17\%) casos foram classificados como criptogênicos e 58 (83\%) sintomáticos, através da utilização de dados semiológicos e exames de neuroimagem (TC e/ou RM de crânio). Antigamente, $40 \%$ dos casos de SW eram classificados como criptogênicos. Atualmente, a disponibilidade de técnicas de neuroimagem sofisticadas têm baixado esta proporção. Chugani e Conti ${ }^{13}$ avaliaram 140 crianças com SW (média de idade de 17 meses) e observaram que, após a realização do PET (positron emission tomography) o número de casos sintomáticos aumentou dramaticamente de $42(30 \%)$ para $134(95,7 \%)$.

Entre os casos sintomáticos do nosso estudo, $15(25,9 \%)$ eram secundários a causas prénatais, $16(27,6 \%)$ perinatais, $9(15,5 \%)$ pós-natais e $18(31 \%)$ de etiologia desconhecida. A síndrome hipóxico-isquêmica, as malformações cerebrais e as infecções do sistema nervoso central foram as etiologias conhecidas mais frequentes. A maioria dos estudos relata que 20 a $30 \%$ dos casos são decorrentes de fatores pré-natais, $15 \%$ a $56 \%$ perinatais e $8 \%$ a $14 \%$ pós-natais ${ }^{14-16}$.

Segundo Lombroso ${ }^{10}$, Glaze e Zion ${ }^{17}$ e Hrachovy e Frost ${ }^{18}$, a probabilidade de desenvolvimento mental normal nos pacientes com SW é relativamente rara, variando de 11 a $29 \%$. Entre os casos sintomáticos, a incidência de desenvolvimento mental normal é baixa, variando de $5 \%{ }^{18}$ a $19 \%{ }^{19}$. Nos criptogênicos a probabilidade é maior, variando de $30 \%$ a $70 \%{ }^{10}$. No presente estudo houve percentagem significativamente maior de pacientes no grupo criptogênico que frequentavam escola regular.

Em nosso estudo, após período de seguimento médio de 6,8 anos, 16 (22,9\%) dos 70 pacientes estavam completamente livres de crises, 16 (22,9\%) evoluíram para síndrome de Lennox-Gastaut, $18(25,7 \%)$ persistiram com espasmos e $20(28,5 \%)$ apresentavam outros tipos de crises (tônicoclônicas generalizadas, tônicas, mioclônicas, parciais simples ou complexas e ausências). No grupo criptogênico houve tendência maior para o controle completo das crises e tendência menor de evolução para a síndrome de Lennox-Gastaut. Riikonen ${ }^{20}$ estudou 214 pacientes com SW que foram seguidos por 20 a 35 anos. Após este período de seguimento, apenas 147 pacientes ainda estavam vivos. Destes, 48 (33\%) estavam livres de crises há mais de 2 anos, 40 (27\%) apresentavam crises parciais, 26 (18\%) evoluíram para síndrome de Lennox-Gastaut, 19 (13\%) não foram classificados e em 14 os dados foram insuficientes. Esta autora também observou que os espasmos cessaram antes dos 2 anos de idade em 65\% dos casos e persistiram após os 3 anos em 4 dos 147 pacientes.

A terapia universalmente reconhecida como a mais eficaz no tratamento da SW é o ACTH. Onze $(68,75 \%)$ dos 16 de nossos pacientes que receberam ACTH, como droga de primeira escolha no tratamento dos espasmos, apresentaram resposta excelente, sem recidiva. Segundo Aicardi ${ }^{21}$, Baram $^{22}$ e $\mathrm{Snead}^{23}, 50$ a $80 \%$ dos pacientes com SW respondem ao tratamento com ACTH. As percentagens variam entre os diferentes estudos em relação à etiologia (melhor resposta entre os casos criptogênicos), dose e duração do tratamento.

Apenas 5 de nossos pacientes receberam VGB como droga de primeira escolha no tratamento dos espasmos. No entanto, apesar do pequeno número da amostra, observamos que, estatisticamente, a eficácia desta droga foi similar a do ACTH, além de apresentar uma menor tendência a recidivas.

A VGB tem se mostrado promissora para o tratamento dos EI, principalmente nos casos associados a esclerose tuberosa. Vários estudos ${ }^{24-29}$ têm relatado o controle de 50 a $73 \%$ dos espasmos 
quando a VGB é utilizada como monoterapia no tratamento da SW. Os efeitos colaterais desta droga parecem ser raros e geralmente leves, tais como sonolência, insônia, hipercinesia ou hipotonia ${ }^{8}$. No entanto, desde junho de 1997, anormalidades do campo visual têm sido relatadas em pacientes tratados com $\mathrm{VGB}^{30-31}$. Assim, a eletrorretinografia e mais recentemente a eletro-oculografia têm sido sugeridas como exames complementares que deveriam ser realizados durante o tratamento com esta droga. A associação da VGB com outras drogas que aumentam os níveis de GABA (por exemplo, o VPA) parece aumentar o risco de toxicidade sobre a retina ${ }^{31}$.

Concluímos que a SW criptogênica, quando comparada à sintomática, apresenta melhor evolução do desenvolvimento psicomotor, da epilepsia e das alterações eletrencefalográficas. No entanto, ainda assim, na maioria dos casos o prognóstico é grave, com prejuízo intelectual e motor. $\mathrm{O}$ ACTH e a VGB, tanto quando usadas como drogas de primeira ou de segunda escolha, foram as mais eficazes no controle dos espasmos, o que torna a última droga uma possível terapia de primeira escolha para o tratamento desta desordem.

Também foi observado que, após a administração de 2 drogas, a introdução de uma terceira ou quarta droga se mostra de pouca eficácia na tentativa do controle dos espasmos.

\section{REFERÊNCIAS}

1. West WJ. One peculiar form of infantile convulsions. Lancet 1841;1:724-725.

2. Gibbs FA, Gibbs EL. Atlas of electroencephalography. Vol 2. Epilepsy. Cambridge: Addison-Wesley, 1952.

3. Sorel L, Dusaucy-Bauloye A. A propor de cas d'hypsarrhytmia de Gibbs: son traitement spectulaire par ACTH. Acta Neurol Belg 1958;58:130-141.

4. Commission on the Classification and Terminology of the International League Against Epilepsy. Proposal for revised classification of epilepsies and epileptic syndromes. Epilepsia 1989;30:389-399.

5. Chiron C, Dulac O, Beaumont D, Palacios L, Pajot N, Mumford J. Therapeutic trial of vigabatrin in refractary infantile spasms. J Child Neurol 1991;6:2552-2559.

6. Appleton RE. The role of vigabatrin in the management of infantile epileptic syndromes. Neurology 1993;43(Suppl 5):S21-S23.

7. Del Giudice E, Latte F, Romano A. Vigabatrin in childhood epileptic syndromes (Abstr). Seizure 1992;1(Suppl A):7-21.

8. Aicardi J, Sabril IS. Investigator and Peer Review Groups, Mumford JP, et al. Vigabatrin as initial therapy for infantile spasms: a European retrospective survey. Epilepsia 1996:37:638-642.

9. Lefévre AB. Exame neurológico evolutivo. 2Ed. São Paulo: Sarvier, 1976,5:1-182.

10. Lombroso CT. A prospective study of infantile spasms: clinical and therapeutic correlations. Epilepsia 1983;24:135-158.

11. Hrachovy RA, Frost JD Jr, Kellaway P. Hypsarrhythmia: variation on the theme. Epilepsia 1984;25:317-325.

12. Cowan LD, Hudson LS. The epidemiology and natural history of infantile spasms. J Child Neurol 1991;6:355-364.

13. Chugani HT, Conti JR. Etiologic classification of infantile spasms in 140 cases: role of positron emission tomography. J Child Neurol 1996;11:44-48.

14. Kurokawa T et al. West syndrome and Lennox-Gastaut syndrome: a survey of natural history. Pediatrics 1980;65:81-88.

15. Walther B, Schimitt T, Reitter B. Identification of infants at risk for infantile spasms bu neonatal polygraphy. Brain Dev 1987;9:377-390.

16. Hughes JR, Long KJ. Infection as possible etiology of hypsarrhytmia. Clin Electroencephalogr 1986;17:78-81.

17. Glaze DG, Zion TE. Infantile spasms. Curr Probl Pediatr 1985;15:1-39.

18. Hrachovy RA, Frost JD. Infantile spasms. Pediatr Clin N Am 1989;36:311-329.

19. Ohtahara S. Transition between syndromes: developmental aspects of epilepsy. Adv Epileptol 1987;16:171-174.

20. Riikonen R. Long-Term outcome of West syndrome: a study of adults with a history of infantile spasms. Epilepsia 1996;37:367-372.

21. Aicardi J. Infantile spasms and related syndromes. In Aicardi J. Epilepsy in children. New York: Raven Press 1986:17-38.

22. Baram TZ. Pathophysiology of massive infantile spasms: perspective on the role of the brain adrenal axis. Ann Neurol 1993;33:231-237.

23. Snead OC III. Treatment of infantile spasms. Pediatr Neurol 1990;6:147-150.

24. Appleton RE, Montiel-Viesca F. Vigabatrin in infantile spasms: why add on ? Lancet 1993;342:962.

25. Vles JSH, Van der Heyden AMHG, Ghjis A, et al. Vigabatrin in the treatment of infantile spasms. Neuropediatrics 1993;24:230-231.

26. Schmitt B, Wohlrab G, Boltshauser E. Vigabatrin in newly diagnosed infantile spasms. Neuropediatrics 1994;24:54.

27. Buti D, Rota M, Lini M, et al. First-line monotherapy with vigabatrin in infantile spasms: long-term clinical and EEG evolution in 12 patients. Epilepsia 1995;36(Suppl 3):S102.

28. Granstrom ML, Gaily E, Lindhl E. Vigabatrin as the first drug in infantile spasms. Epilepsia 1995;36(Suppl 3):S102.

29. Chiron C, Dumas C, Dulac O, et al.. Vigabatrin versus hydrocortisone as first-line monotherapy in infantile spasms due to tuberous sclerosis. Epilepsia 1995;36(Suppl3):S265.

30. Harding GF. Severe persistent visual field constriction associated with vigabatrin: four possible explanations exist. BMJ 1997;314:1694-5. Letter.

31. Arndt CF, Derambure P, Deffort-Dhellemmes S, Hache JC. Outer retinal dysfunction in patients treated with vigabatrin. Neurology 1999;52:1201-1205. 